УДК $535.662 .2+778.68$

DOI: https://doi.org/10.17308/kcmf.2019.21/769

Поступила в редакцию 03.04.2019

Подписана в печать 15.05.2019

\title{
ВЛИЯНИЕ ТЕРМИЧЕСКОГО И АНИОННО-ПРИМЕСНОГО ФАКТОРОВ НА ЭЛЕКТРОПОЛЕВУЮ КОАЛЕСЦЕНЦИЮ АТОМОВ СЕРЕБРА В МИКРОКРИСТАЛЛАХ ЕГО ГАЛОГЕНИДОВ
}

\author{
(c) 2019 О. Н. Шишканов, А. П. Бойченко \\ Кубанский государственный университет \\ ул. Ставропольская, 149, 350040 Краснодар, Российская Федерация
}

\begin{abstract}
Аннотация. На примере фотоэмульсионных микрокристаллов галогенидов серебра математически описана зависимость коалесценции атомов серебра под действием неоднородного электрического поля от температуры окружающей среды и концентрации содержащихся в микрокристаллах примесей анионов йода. Показана корреляция экспериментальных данных по электрополевой чувствительности на примере промышленно выпускаемых фотоматериалов и специально изготовленных с результатами физико-математического моделирования коалесцентно-электрополевого процесса. Установлено, что при малом изменении температуры в 6 K (относительно стандартной в 298 К) и естественном влагосодержании желатины 7.5 \% происходит быстрый рост поверхностных атомов Ag. Такой же эффект достигается путем замещения в микрокристаллах $\mathrm{AgBr}$ доли анионных примесей йода до величины порядка 5 \% от доли ионов брома при температуре $298 \mathrm{~K}$.
\end{abstract}

Ключевые слова: электрополевое изображение, электрополевая чувствительность, галогениды серебра, коалесценция атомов и кластеров серебра, термовлияние, анионные примеси.

\section{ВВЕДЕНИЕ}

В 1970 году авторами [1-3] был обнаружен эффект образования проявляемых центров скрытого изображения (ЦСИ) в галогенсеребряных (AgHal) фотоэмульсиях под действием неоднородного электрического поля без какоголибо освещения. Благодаря способности выявлять малейшие изменения топографии электрополевой неоднородности эффект получил название «электротопографический» (ЭТГ). Тогда же был предложен возможный механизм ЭГТ-эффекта [1-3]. В его основу первооткрывателями положена существовавшая в то время термодинамическая теория фотографического процесса [4], которую, по их мнению, ЭТГ-эффект подтверждал. Позднее, на основе концепции светочувствительности К. В. Чибисова о существовании в микрокристаллах (MK) AgHal первичных и вторичных серебряных (Ag) центров - центров проявления (также включающих ЦСИ) [5], рассматривалась их термическая устойчивость

$\triangle$ Бойченко Александр Павлович, e-mail: bojchenco@yandex.ru с указанием на неудачливость названия «термодинамическая теория» [6]. Термическую ассоциацию Ag-кластеров с образованием более крупных агрегатов и распад уже возникших ЦСИ путем отщепления от них отдельных атомов или малоатомных кластеров Ag авторы формально уподобляли каплям жидкости, находящейся в контакте с паром, от чего описываемый процесс ими назван «коалесцентным» [6]. Особая роль в нем уделялась локализованным на различного рода ловушках (особенно поверхностных) фотоэлектронам, в неоднородном электрическом поле которых осуществляют свое движение поляризованные атомы Ag и/или их малоатомные кластеры, преодолевая диполь-дипольное отталкивание и устремляясь в сторону наибольшего электрополевого градиента, где и образуют в итоге устойчивый Ag-агрегат или ЦСИ. На основе этих рассуждений авторы приходят к заключению об отсутствии принципиальных отличий между способами создания такого поля: будет ли оно создано локализованным электроном или каким-либо внешним источником [6]. Раз- 
ница состоит лишь в масштабах времени, расстояния, напряженности электрического поля и степени его неоднородности [6]. Таким образом, сущность ЭТГ-эффекта заключается в принудительной коалесценции (слипании) атомов $\mathrm{Ag}$ и образовании из них проявляемых ЦСИ на какой-то определенной части MK AgHal, куда их стягивает внешнее неоднородное электрическое поле или поле индуцированных им зарядов межфазной (например, на границе раздела между МК и желатиной фотоэмульсии) поляризации $[1-3,6]$. Именно неоднородность поля оказывает решающее значение в формировании Ag-центров электрополевого изображения (ЭПИ), что экспериментально доказано авторами ЭТГ-эффекта на примере ЭПИ металлической сеточки и сферического электрода, изображение которого имеет форму кольца [1, 2]. Так, в центре ЭПИ, где сферический электрод контактирует с AgHal-фотоэмульсией, напряженность поля (включая и создаваемый им ток имеющихся носителей заряда) максимальна, но оно там однородно, поэтому изображение в центре отсутствует (изображение на уровне плотности оптической вуали). На периферии же электрода поле минимально, но имеет убывающий градиент, благодаря чему и формируется кольцевая форма ЭПИ такого или геометрически подобных ему электродов [1, 2].

Из вышеизложенного ясно, что ЭТГ-эффект оказывается крайне чувствительным к градиентным изменениям электрического поля. Это позволило его первооткрывателям разработать технологию электрополевой дефектоскопии, с успехом использованной на орбитальной станции «Салют-7» для выполнения ряда исследований по динамике деградации модельных и конструкционных материалов под действием физических факторов космического пространства [7]. Несмотря на то, что позднее названный эффект обнаружился на некоторых электретах (живичной канифоли и сополимера стирола с октилметакрилатом [2]), MK AgHal остаются в настоящее время пока не превзойденными по своей чувствительности к электрополевому воздействию. Как установлено в $[1,2]$ и нашими исследованиями [8, 9], это свойство определяется не только размерами, формой и степенью химической (восстановительной) сенсибилизации MK AgHal, но и определенной сбалансированностью $\Theta$ удельных электропроводностей фотоэмульсионного слоя $\sigma_{f}$, содержащего кристаллики, и подложки фотоматериала $\sigma_{p}$, которая при наличии тонкого воздушного про- межутка над фотослоем подчиняется условию: $0.09 \leq \Theta=\sqrt{\frac{\sigma_{p} \varepsilon_{f}}{\sigma_{f} \varepsilon_{p}}} \leq 0.23$, где $\varepsilon_{f}$ и $\varepsilon_{p}-$ диэлектрические проницаемости вышеназванных слоев. Такое условие баланса способствует эффективной локализации объемного электрического заряда межфазной поляризации в AgHal-слое. Как уже было сказано, под действием градиента созданного им электрического поля в МК и формируются Ag-ЦСИ [1-3]. Физико-математическая модель кинетики протекания этого процесса впервые предложена в нашей работе [10]. Из нее следует ряд научно важных и практически значимых результатов: в зависимости от термодинамических, электрофизических, химических факторов, а также в особенности от влагосодержания желатины, определяющую роль процесса коалесценции могут играть как поверхностные, так и глубинные атомы Ag. Например, при всех прочих равных условиях наибольшая электрополевая чувствительность (ЭПЧ) MK AgHal может достигаться при уменьшении температуры $T$ (относительно стандартной $T_{R}=298 \mathrm{~K}$ ) или замещением в кристалликах ионов брома $\left(\mathrm{Br}^{-}\right)$ионами йода $\left(\mathrm{I}^{-}\right)$, чего ранее в механизме ЭТГ-эффекта не рассматривалось [1-3, 6]. Предложенная модель допускает вариацию многих параметров, влияющих на эффективность электрополевой коалесценции атомов Ag при формировании ЭПИ. Поэтому настоящая статья посвящена экспериментальной проверке некоторых результатов моделирования, представляющих практический интерес для электрополевой визуализации на AgHal-фотоматериалах, у которых при определенном влагосодержании желатины и разнодолевом содержании MK AgHal в фотоэмульсии $P_{k}$ возможно существенное повышение ЭПЧ как путем малого изменения $T$, так и введением в MK AgBr малых концентраций ионов $\mathrm{I}^{-}$, замещающих ионы $\mathrm{Br}^{-}$.

\section{ТЕОРЕТИЧЕСКАЯ ЧАСТЬ}

Для физико-математического описания кинетики процессов формирования ЭПИ из атомов Ag рассматривался MK AgBr микронных размеров радиусом $r$ с варьируемой в нем долей $\alpha$ примеси ионов I- от 0 до 30 \%. При этом учитывались атомы Ag, группируемые полем не только на поверхности MК, но и в их глубине, а также концентрационная кинетика глубинных атомов $\mathrm{Br}$ и I. К перечисленным атомам в рассмотрение включены их неподвижные ионы $\mathrm{Ag}_{0}^{+}, \mathrm{Br}_{0}^{-}, \mathrm{I}_{0}^{-}$, образующие решетку кристалли- 
ка, и подвижные, создающие в нем катионные дефекты $V_{\mathrm{Ag}}$ из ионов $\mathrm{Ag}^{+}$и анионные $V_{\mathrm{Br}}$ и $V_{\mathrm{I}}-$ из ионов $\mathrm{Br}^{-}$и I'-, а также темновые электроны $e$ и дырки $h$, совместно образующиеся в процессе синтеза и химической сенсибилизации таких МК. В модели также учитывался характер распределения напряженности электрического поля на границе раздела МК и желатиновой матрицы в зависимости от ее удельной электропроводности $\sigma_{G}=e\left(n_{\mathrm{v}+} \mu_{\mathrm{v}+}+n_{\mathrm{v}-} \mu_{\mathrm{v}-}\right) \exp \left\{\frac{e a_{G} E_{f}}{2 k T}\right\}$ и диэлектрической проницаемости $\varepsilon_{G}=\varepsilon_{0 G}\left(1+P_{\mathrm{v}} A^{-1}\right)$, где $\varepsilon_{0 G}-$ проницаемость безводной желатины; $A=\left(1-P_{\mathrm{v}}\right) \cdot 3^{-1}+\varepsilon_{0 G} \cdot\left(\varepsilon_{\mathrm{v}}-\varepsilon_{0 G}\right)^{-1}$; диэлектрическая проницаемость воды $\varepsilon_{\mathrm{v}}$ с ее объемной долей $P_{\mathrm{v}}$ в желатине; $a_{G}=1.35 \cdot 10^{-9} \mathrm{~m}$ - диаметр молекулы желатины; $\mu_{v+}, \mu_{\mathrm{v}-}$ и $n_{\mathrm{v}+}=n_{\mathrm{v}-}=\sqrt{1.12 \cdot 10^{11} P_{\mathrm{v}} B \exp (-C)}-$ соответст венно подвижности и начальная концентрация ионов гидроксония и гидроксила, равновесно образующихся в биополимере при диссоциации адсорбированных им молекул воды диаметром $a_{\mathrm{v}}$, молекулярной массой $M_{\mathrm{v}}$ и плотностью воды $\rho_{\mathrm{v}} ; B=\rho_{v} N_{A}\left(M_{v} \cdot 10^{-3}\right)^{-1}$; $C=e^{2}\left(8 \pi k T \varepsilon_{0} \varepsilon_{v} a_{v}\right)^{-1} ; \varepsilon_{0}$ - диэлектрическая постоянная; $k$ и $N_{\mathrm{A}}$ - постоянные Больцмана и Авогадро; $e$ - элементарный электрический заряд; $E_{f}=\frac{\varepsilon_{p} \cdot U}{d_{f} \varepsilon_{p}+d_{p} \varepsilon_{f}}-$ напряженность электрического поля в фотоэмульсии толщиной $d_{f}=5 \cdot 10^{-6} \mathrm{~m}$ и $\varepsilon_{f}=Q+\sqrt{Q^{2}+0.5 \varepsilon_{G} \varepsilon_{k}}$, нанесенной на полимерную подложку толщиной $d_{p}=1.65 \cdot 10^{-4} \mathrm{~m}$ и диэлектрической проницаемостью $\varepsilon_{p}=3.23$; $Q=0.25\left[\left(3 P_{G}-1\right) \varepsilon_{G}+\left(3 P_{k}-1\right) \varepsilon_{k}\right]$, где $\varepsilon_{k}-$ диэлектрическая проницаемость $\mathrm{MK} \mathrm{AgBr}$ и $P_{G}$ - доля желатины в фотоэмульсии.

Как правило, для формирования Ag-центров скрытого ЭПИ достаточно одиночного импульса электрического поля с $E_{f} \approx 10^{5} \mathrm{~V} / \mathrm{m}$ [9] и длительностью $\tau \geq 0.1 \mathrm{~s}$ [8]. Поэтому для моделирования был выбран часто встречающийся и технологически просто реализуемый видеоимпульс напряжения $U$ колоколообразной формы, описываемый уравнением вида [10]:

$$
\begin{aligned}
& U=N_{U} U_{0}\left[\exp \left(-\frac{\tau}{\tau_{1}}\right)+\exp \left(-\frac{\tau}{\tau_{2}}\right)\right] \times \\
& \times\left[\exp \left(-\frac{\tau}{\tau_{2}}\right)-\exp \left(-\frac{\tau}{\tau_{1}}\right) \cdot \frac{\left(\tau+\tau_{1}\right) \tau_{2}}{\left(\tau+\tau_{2}\right) \tau_{1}}\right],
\end{aligned}
$$

где $N_{U}=1.26$ - поправочный коэффициент; $U_{0}$ - начальное напряжение импульса, выбранное

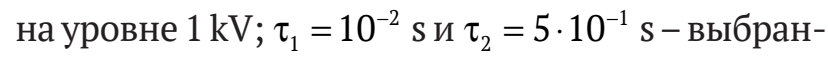
ные для моделирования длительности его переднего и заднего фронтов.

Согласно [2, 11], при указанной величине $\tau_{1} \leq 10^{-2}$ s существенно проявление релаксационных процессов поля как в объеме MK AgHal $E_{k}$, так и на его поверхности $E_{s k}$. Поэтому с учетом экспериментально исследованных систем MK AgBr-желатина (фотоэмульсий) [11] уравнения для $E_{k}$ и $E_{s k}$ записывались в виде:

$$
\begin{gathered}
E_{k}=3 E_{k}^{\prime}-3 \tau_{k}^{-1} \exp \left(-\tau \tau_{k}^{-1}\right) \cdot \int_{0}^{\tau} E_{k}^{\prime} \exp \left(\tau \tau_{k}^{-1}\right) d \tau \\
E_{s k}=3 E_{f} \sqrt{K}-3 \tau_{f}^{-1} \exp \left(-\tau \tau_{f}^{-1}\right) \int_{0}^{\tau} E_{f} \sqrt{K} \exp \left(\tau \tau_{f}^{-1}\right) d \tau
\end{gathered}
$$

где

$$
\begin{gathered}
K=\left[\sigma_{G}^{2}+\left(2 \pi \tau_{f}^{-1}\right)^{2} \varepsilon_{G}^{2} \varepsilon_{0}^{2}\right] \times \\
\times\left[\left(\sigma_{k}+2 \sigma_{G}+2 \sigma_{S k} \cdot r^{-1}\right)^{2}+\left(2 \pi \tau_{f}^{-1}\right)^{2} \varepsilon_{0}^{2}\left(\varepsilon_{k}+2 \varepsilon_{G}\right)^{2}\right]^{-1} ;
\end{gathered}
$$

$\tau_{k}=\varepsilon_{0} \varepsilon_{k} \sigma_{k}^{-1}$ и $\tau_{f}=\varepsilon_{0} \varepsilon_{f} \sigma_{f}^{-1}-$ время ионной релаксации MK AgHal и фотоэмульсии.

Для упрощения записи кинетических уравнений, входящие в них параметры систематизировались путем присвоения каждой частице порядкового номера, в совокупности выраженных через обобщенный индекс $i$, а варианты комбинаций возможных взаимодействий частиц друг с другом - через вспомогательные индексы $j, l, \eta$ и $q$. Расшифровка индексов параметров приведена в табл. 1. Тогда с учетом изложенного, некоторые макропараметры, выражающиеся через микропараметры, примут вид: удельная объемная электропроводность MK AgBr $\sigma_{k}=\left(e Z \sum_{i=1}^{8} n_{0 i} \mu_{i}\right) \exp \left\{\frac{e a_{k} E_{f}}{2 k T}\right\}$

и их поверхностная электропроводн о с т ь $\quad \sigma_{S k}=\left(e Z R_{d} \sum_{i=1}^{8} n_{0 i} \mu_{i}\right) \exp \left\{\frac{e a_{k} E_{f}}{2 k T}\right\}, \quad$ г д е $R_{d}=\sqrt{\varepsilon_{k} \varepsilon_{0} k T e^{-2} Z^{-2}\left(\sum_{i=1}^{8} n_{0 i}\right)^{-1}}$ - дебаевский радиус

МК с кратностями заряда входящих в него подвижных ионов $Z=1$, радиусом $r_{i}$ и их начальной концентрацией $n_{01-06}=\sqrt{\frac{r}{r_{1-6}}} a_{k}^{-3} \exp \left\{-\frac{W_{f}}{2 k T}\right\}$, рав-

новесно образующихся со своими вакансиями при рассматриваемой $T$ в диапазоне 273-313 К и энергией протекания этого процесса по Я. Френкелю $-W_{f}=1.7 \cdot 10^{-19}-1.1 \cdot 10^{-18} k T$ J [12]. При этом математическая запись для $\sigma_{f}$ примет вид: 
Таблица 1. Расшифровка индексов параметров, входящих в уравнения (4)-(9)

[Table 1. Interpretation of indices of parameters included in equations (4)-(9)]

\begin{tabular}{|c|c|c|c|c|c|}
\hline \multirow{2}{*}{$\begin{array}{c}\text { Параметр } \\
\text { [Parameter] }\end{array}$} & \multicolumn{5}{|c|}{$\begin{array}{c}\text { Значение индекса } \\
\text { [Index value] }\end{array}$} \\
\hline & $i$ & $j$ & $l$ & $\eta$ & $q$ \\
\hline$V_{\mathrm{Ag}}$ & 1 & - & $9-11,13$ & 1 & - \\
\hline $\mathrm{Ag}^{+}$ & 2 & - & $9-12,14,15$ & 2 & - \\
\hline $\mathrm{Br}^{-}$ & 3 & - & $9-11,13$ & 3 & - \\
\hline$V_{\mathrm{Br}}$ & 4 & - & $9-11,14,15$ & 4 & - \\
\hline $\mathrm{I}^{-}$ & 5 & - & $9-11,13$ & 5 & - \\
\hline$V_{\mathrm{I}}$ & 6 & - & $9-11,14,15$ & 6 & - \\
\hline$e$ & 7 & - & $9-13$ & 7 & - \\
\hline$h$ & 8 & - & $9-12,14,15$ & 8 & - \\
\hline $\mathrm{Ag}$ & 9 & $1-11$ & 10,11 & - & 9 \\
\hline $\mathrm{Br}$ & 10 & $1-11$ & 9,10 & - & 10 \\
\hline $\mathrm{I}$ & 11 & $1-11$ & 9,11 & - & 11 \\
\hline $\mathrm{Ag}_{\mathrm{s}}$ & 12 & $2,7,8,12$ & - & - & 12 \\
\hline $\mathrm{Ag}_{0}^{+}$ & 13 & - & - & - & - \\
\hline $\mathrm{Br}_{0}^{-}$ & 14 & - & - & - & - \\
\hline $\mathrm{I}_{0}^{-}$ & 15 & - & - & - & - \\
\hline
\end{tabular}

$\sigma_{f}=G+\sqrt{G^{2}+0.5 \sigma_{G} \sigma_{k}}$, где $G=0.25\left[\left(3 P_{G}-1\right) \sigma_{G}+\right.$ $\left.+\left(3 P_{k}-1\right) \sigma_{k}\right]$, a $E_{k}^{\prime}=U\left[\left(2+\varepsilon_{k} \varepsilon_{f}^{-1}\right)\left(d_{f}+d_{p} \varepsilon_{f} \varepsilon_{p}^{-1}\right)\right]^{-1}$ - мгновенная напряженность поля в МK, когда $\tau_{k} \gg \tau$ [11]. Начальные концентрации для электронов и дырок записывались в виде: $n_{07}=n_{08}=\left(n_{03}+n_{05}\right) \exp \left\{-\frac{W_{e}}{2 k T}\right\}$ (прич е м,$\left.\quad n_{05}=\alpha n_{03}\right), \quad$ а $\quad n_{09}=n_{013} n_{07} k_{1} \exp \left\{-\frac{W_{\mathrm{Ag}}}{2 k T}\right\}$ и $n_{010,011}=n_{03,05} \exp \left\{-\frac{W_{e}}{2 k T}\right\}-$ для соответствующих атомов, где $k_{1}=\frac{4}{3} \pi L_{a}^{3}-$ константа реакции межатомного взаимодействия, в которой $L_{a}=2.6055 \cdot 10^{-9} \mathrm{~m}$ - расстояние взаимодействия между ионами $\mathrm{Ag}^{+}$внутри $\mathrm{MK} \mathrm{AgBr}$, теоретически определенное в [13];

$$
\begin{gathered}
n_{012}=n_{013} k_{2}\left[n_{07} \exp \left\{-\frac{W_{\mathrm{sAg}}}{2 k T}\right\}+n_{\mathrm{s}} \exp \left\{-\frac{W_{\mathrm{S}}}{2 k T}\right\}+\right. \\
\left.+n_{\mathrm{Au}} \exp \left\{-\frac{W_{\mathrm{Au}}}{2 k T}\right\}\right]
\end{gathered}
$$

- начальная концентрация поверхностных атомов $\mathrm{Ag}_{\mathrm{s}}$, где $k_{2}=\frac{4}{3} \pi R_{d}^{3}-$ константа реакции вза- имодействия между неподвижными ионами $\mathrm{Ag}_{0}^{+}$ с электронами и другими частицами-сенсибилизаторами в поверхностном слое $\mathrm{MK} \mathrm{AgBr}$, а $n_{\mathrm{S}}$ и $n_{\mathrm{Au}}-$ концентрации вводимых ионов серы и золота при сернисто-золотой сенсибилизации; $W_{\mathrm{s}}$ и $W_{\mathrm{Au}}$ - энергии адсорбции серы [14] и золота [15]. На основе данных [16] в диапазоне рассматриваемых $T$ и $\alpha$ с помощью полиномов выведена функциональная зависимость постоянной кристаллической решетки MK AgBr от этих параметров: $a_{k}=\left(5.77476 \cdot 10^{-10}+\alpha_{T} \cdot \alpha\right) \cdot \sqrt[3]{\Psi}$, где $\Psi=\left(1+3 \alpha_{\mathrm{k}}+3 \alpha_{\mathrm{k}}^{2} \cdot\left(T-T_{R}\right)+\alpha_{\mathrm{k}}^{3} \cdot\left(T-T_{R}\right)^{2}\right) \cdot\left(T-T_{R}\right), \mathrm{a}$ $T_{R}=298 \mathrm{~K}, \alpha_{T}=2.57226(6) \cdot 10^{-13} \mathrm{~m}$ и $\alpha_{\mathrm{k}}=3.37 \cdot 10^{-5} \mathrm{~K}^{-1}$ - коэффициенты. Следует отметить, что в рассматриваемой модели $\varepsilon_{k}$ является функцией от $\alpha$ и $T$, а $\varepsilon_{v}$ - от последнего параметра. Математическая запись функциональных зависимостей для перечисленных параметров подробно рассмотрена в нашей обобщенной работе [17].

Вышеизложенное позволяет записать для всех рассматриваемых частиц MK AgBr систему одномерных кинетических уравнений непрерывности, справедливых для кристалликов микронных размеров [10, 12], совместно с уравнением Пуассона и соответствующими граничными условиями:

$$
\frac{\partial n_{i}}{\partial t}=-\frac{\partial J_{i}}{\partial x}+\sum_{j} P_{i j} n_{j}-n_{i} \sum_{l} P_{l i}
$$




$$
\begin{gathered}
J_{i}=-D_{i} \frac{\partial n_{i}}{\partial x} \pm \mu_{\eta} n_{\eta} \frac{\partial \varphi}{\partial x}+\frac{F_{q}^{\prime} n_{q}}{m_{q} \gamma_{q}} ; \\
\frac{\partial^{2} \varphi}{\partial x^{2}}=\frac{e Z}{\varepsilon_{k} \varepsilon_{0}}\left(\sum_{v=2(\beta+1)} n_{v}-\sum_{\rho=2 \beta+1} n_{\rho}\right), \beta=0,1,2,3 ; \\
n_{i}(0, t)=n_{0 i} \exp \left( \pm \frac{\mu_{\eta} E_{k} R_{d}}{D_{\eta}}-\frac{F_{q}^{\prime} R_{d}}{k T}\right) ; \\
=n_{0 i}\left(\mp \frac{\mu_{\eta} E_{k}}{D_{\eta}}+\frac{F_{q}^{\prime}}{k T}\right) \exp \left( \pm \frac{2 \mu_{\eta} E_{k}\left[R_{d}-r\right]}{D_{\eta}}-\frac{2 F_{q}^{\prime}\left[R_{d}-r\right]}{k T}\right) ; \\
F_{q}^{\prime}=\left\{\begin{array}{c}
\frac{\partial n_{i}(r, t)}{\partial r}= \\
F_{s}=e E_{s k}-\frac{E_{k} d_{q}}{a_{k}}, \quad q=12 ; \\
F=\frac{E_{k} d_{q}}{a_{k}}, \quad q \neq 12,
\end{array}\right.
\end{gathered}
$$

где $n_{i}$ - текущие концентрации частиц, участвующих в процессе коалесценции; $J_{i}$ - потоки этих частиц; $P_{i j}=\pi n_{0 i} r_{i}^{2} v_{j}-$ вероятности захвата $i$-ой частицей $j$-ой частицы, а $P_{l i}=\pi n_{01} r_{l}^{2} v_{i}-$ вероятности захвата $l$-ой частицей $i$-ой частицы. Причем, для неподвижных ионов кристаллической решетки $(i=13-15)$ с кратностями их зарядов $Z_{x}=1$, кинетические уравнения отсутствуют, а $r_{13-15}=\frac{e^{2} Z Z_{x}}{\varepsilon_{0} \varepsilon_{k} k T} \quad[16]$. Па раме тры $D_{1-8}=$ $=\frac{v_{1-8} n_{01-08}^{-1 / 3}}{3} \exp \left(-\frac{W_{k}}{k T}\right)$ и $\mu_{1-8}=\frac{e Z D_{1-8}}{k T}-$ диффузии и подвижности частиц массами $m$ со скоростями их теплового движения $v=\sqrt{\frac{3 k T}{m}} ; W_{k}=\frac{\alpha_{M} e^{2} Z_{x}^{2}}{4 \pi \varepsilon_{k} \varepsilon_{0} a_{k}}$ - потенциальная энергия взаимодействия ионов кристаллической решетки $\mathrm{AgBr}$ с ее постоянной Маделунга $\alpha_{M}=1,7476$ [16]; $F_{q}^{\prime}$ - силы, действующие на соответствующие атомы с их дипольными моментами $d_{q}$ и коэффициентами диффузии $D_{9-12}=\frac{v_{9-12} a_{k}}{3} \exp \left(-\frac{W_{k}}{k T}\right) ; \gamma_{q}=\frac{k T}{m_{q} D_{q}}-$ коэффициент сопротивления среды; $\varphi$ - потенциал, создаваемый всеми подвижными электрически заряженными частицами MK AgHal; $x$ и $t$ - текущие координата и время. В табл. 2 приведены используемые при численном решении системы названия и обозначения физических параметров, часть значений которых с соответствующими значащими цифрами после запятой взята из [6, 13-16, 18]. Отметим, что содержащи- еся в уравнениях (4)-(9) слагаемые, индексы параметров которых отсутствуют в табл. 1 (прочерк), обращаются в нуль. Система уравнений (4)-(9) с начальными условиями для всех рассмотренных выше частиц и граничными условиями (7), (8) численно решалась методом Рунге-Кутта. Для представления результатов моделирования в виде поверхностных и глубинных концентраций атомов Ag, отнесенных к рассматриваемому МК радиусом $r$, его поверхность представлялась сферическим слоем толщиной, равной $R_{d}$ и объемом слоя $V_{s}=V_{k}-V_{0}$, где $V_{k}=\frac{4}{3} \pi r^{3}-$ объем всего МК, а $V_{0}=\frac{4}{3} \pi\left(r-R_{d}\right)^{3}-$ объем его глубинной части. На трехмерных графиках рис. 1 и рис. 2 представлены результаты моделирования для концентраций поверхностных (1) $n_{\text {sAg }}(i=12)$ и глубинных (2) $n_{\mathrm{Ag}}(i=9)$ атомов Ag, образующих под действием импульса электрического поля с $\tau=1 \mathrm{~s}$ центры скрытого ЭПИ в MK AgHal диаметром $10^{-6} \mathrm{~m}$ при различных условиях. Рис. 1 отражает влияние термического и анионно-примесного факторов на этот процесс при $P_{k}=30$ \% и 7.5 \% влагосодержании желатины, а рис. 2 - зависимость этого процесса от долевого содержания $\mathrm{MK} \mathrm{AgBr}$ в фотоэмульсии и ее влагосодержания при $T_{R}=298 \mathrm{~K}$.

Из первого графика (рис. 1) видно, что весьма чувствительными к термическому и анионно-примесному факторам оказываются поверхностные атомы Ag. Причем их максимальная концентрация во время электрополевой коалесценции достигается при 5 \% содержании ионов $\mathrm{I}^{-}$в МК или с понижением температуры от 298 до 292 К. Эффективность этого процесса быстро убывает при уменьшении температуры ниже 288 К. Однако, начиная с этой температуры и повышением концентрации ионов $\mathrm{I}^{-}$от 23 до 30 \%, существенный вклад в коалесцентно-электрополевой процесс вносят глубинные атомы Ag, максимум $n_{\mathrm{Ag}}$ которых приходится на 282 К. В этом случае величина $n_{\text {Ag }}$ оказывается на три порядка больше $n_{\text {sAg }}$, тогда как при $298 \mathrm{~K}$ - на один. Как видно из второго графика (рис. 2), на электрополевую коалесценцию атомов Ag cyщественное влияние оказывает не только влагосодержание желатины, но и концентрация содержащихся в ней МК. На примере беспримесных MK AgBr видно, что максимальная эффективность названного процесса достигается при значениях первого параметра в $\sim 4$ \% и второго - в $5 \%$, т. е. в условиях минимальной концентрации МК в фотоэмульсии и ее расчетной 
Таблица 2. Значения физических и геометрических параметров, выбранных для численных расчетов

[Table 2. The values of physical and geometrical parameters, selected for numerical calculations]

\begin{tabular}{|c|c|c|}
\hline \multicolumn{2}{|l|}{$\begin{array}{l}\text { Параметр, его обозначение и размерность } \\
\text { [Parameter, its designation and dimension] }\end{array}$} & $\begin{array}{l}\text { Значение } \\
\text { [Value] }\end{array}$ \\
\hline $\begin{array}{l}\text { Эффективная масса электрона, kg } \\
\text { [Effective electron mass, kg] }\end{array}$ & $m_{7}$ & $2.642 \cdot 10^{-31}$ \\
\hline $\begin{array}{l}\text { Эффективная масса дырки, } \mathrm{kg} \\
\text { [Effective electron hole mass, kg] }\end{array}$ & $m_{8}$ & $4.555 \cdot 10^{-30}$ \\
\hline Масса атома серебра, kg [Silver atom mass, kg] & $m_{9,12}$ & $1.791 \cdot 10^{-25}$ \\
\hline Радиус атома серебра, m [Silver atom radius, m] & $r_{9,12}$ & $1.44 \cdot 10^{-10}$ \\
\hline Масса иона серебра, kg [Silver ion mass, kg] & $m_{2}$ & $1.777 \cdot 10^{-25}$ \\
\hline $\begin{array}{l}\text { Радиус иона серебра и его вакансии, } \mathrm{m} \\
\text { [Silver ion and vacancy defect radius, m] }\end{array}$ & $r_{1,2}$ & $1.13 \cdot 10^{-10}$ \\
\hline $\begin{array}{l}\text { Масса ионной вакансии серебра, } \mathrm{kg} \\
\text { [Silver ion vacancy defect mass, } \mathrm{kg} \text { ] }\end{array}$ & $m_{1}$ & $2.0 \cdot 10^{-20}$ \\
\hline Масса атома брома, kg [Bromine atom mass, kg] & $m_{10}$ & $1.317 \cdot 10^{-25}$ \\
\hline Радиус атома брома, m [Bromine atom radius, $\mathrm{m}$ ] & $r_{10}$ & $1.14 \cdot 10^{-10}$ \\
\hline Масса иона брома, kg [Bromine ion mass, kg] & $m_{3}$ & $1.331 \cdot 10^{-25}$ \\
\hline $\begin{array}{l}\text { Радиус иона брома и его вакансии, } \mathrm{m} \\
\text { [Bromine ion and vacancy defect radius, } \mathrm{m} \text { ] }\end{array}$ & $r_{3,4}$ & $1.96 \cdot 10^{-10}$ \\
\hline $\begin{array}{l}\text { Масса ионной вакансии брома, } \mathrm{kg} \\
\text { [Bromine ion vacancy defect mass, } \mathrm{kg}]\end{array}$ & $m_{4}$ & $1.5 \cdot 10^{-20}$ \\
\hline Масса атома йода, kg [Iodine atom mass, kg] & $m_{11}$ & $2.107 \cdot 10^{-25}$ \\
\hline Радиус атома йода, $\mathrm{m}$ [Iodine atom radius, m] & $r_{11}$ & $1.33 \cdot 10^{-10}$ \\
\hline Масса иона йода, kg [Iodine ion mass, kg] & $m_{5}$ & $2.121 \cdot 10^{-25}$ \\
\hline $\begin{array}{l}\text { Радиус иона йода и его вакансии, } \mathrm{m} \\
\text { [Iodine ion and vacancy defect radius, } \mathrm{m} \text { ] }\end{array}$ & $r_{5,6}$ & $2.2 \cdot 10^{-10}$ \\
\hline $\begin{array}{l}\text { Масса ионной вакансии йода, } \mathrm{kg} \\
\text { [Iodine ion vacancy defect mass, } \mathrm{kg} \text { ] }\end{array}$ & $m_{6}$ & $2.387 \cdot 10^{-20}$ \\
\hline $\begin{array}{l}\text { Дипольный момент атома серебра, C·m } \\
\text { [Silver atom electric dipole moment, C·m] }\end{array}$ & $d_{9,12}$ & $4.6143 \cdot 10^{-29}$ \\
\hline $\begin{array}{l}\text { Дипольный момент атома брома, C·m } \\
\text { [Bromine atom electric dipole moment, C.m] }\end{array}$ & $d_{10}$ & $3.653 \cdot 10^{-29}$ \\
\hline $\begin{array}{l}\text { Дипольный момент атома йода, C·m } \\
\text { [Iodine atom electric dipole moment, C·m] }\end{array}$ & $d_{11}$ & $4.262 \cdot 10^{-29}$ \\
\hline $\begin{array}{l}\text { Энергия образования электронов и дырок в кристалле AgHal, J } \\
\text { [The energy of formation of electrons and electron hole in an AgHal crystal, J] }\end{array}$ & $W_{e}$ & $2.3315 \cdot 10^{-19}$ \\
\hline $\begin{array}{l}\text { Энергия образования глубинных атомов серебра, J } \\
\text { [The deep atom of silver the formation energy, J] }\end{array}$ & $W_{\mathrm{Ag}}$ & $1.009 \cdot 10^{-21}$ \\
\hline $\begin{array}{l}\text { Энергия образования поверхностных атомов серебра, J } \\
\text { [The surface atom of silver the formation energy, J] }\end{array}$ & $W_{\mathrm{sAg}}$ & $3.365 \cdot 10^{-20}$ \\
\hline $\begin{array}{l}\text { Энергия адсорбции серы на поверхности кристалла AgBr, J } \\
\text { [Sulfur adsorption energies on the surface of an AgBr crystal, J] }\end{array}$ & $W_{\mathrm{s}}$ & $8.01 \cdot 10^{-20}$ \\
\hline $\begin{array}{l}\text { Энергия адсорбции золота на поверхности кристалла AgBr, J } \\
\text { [Gold adsorption energies on the surface of an AgBr crystal, J] }\end{array}$ & $W_{\mathrm{Au}}$ & $6,408 \cdot 10^{-20}$ \\
\hline
\end{tabular}




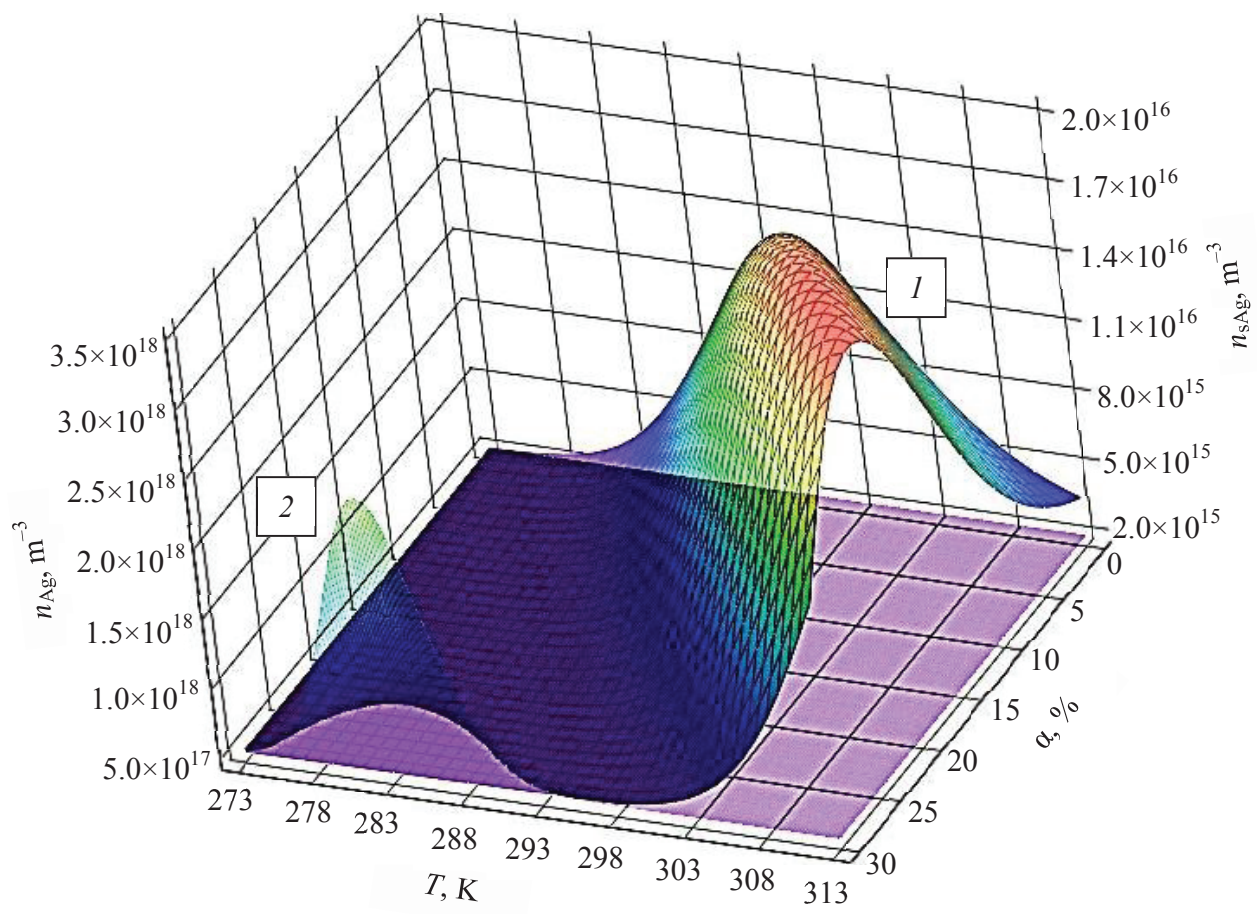

Рис. 1. Изменение концентраций поверхностных (1) и глубинных (2) атомов Ag в MK AgHal в зависимости от температуры $Т$ и доли анионных примесей $\alpha$ в виде ионов $\mathrm{I}^{-}$при $P_{k}=30 \%$ и $P_{\mathrm{v}}=7.5 \%$

[Fig. 1. The changes in the concentrations of surface (1) and deep (2) Ag atoms in AgHal microcrystals as a function of temperature $T$ and the fraction of anionic impurities $\alpha$ in the form of $\mathrm{I}^{-}$ions at the $P_{k}=30 \%$ and at the $\left.P_{\mathrm{v}}=7.5 \%\right]$

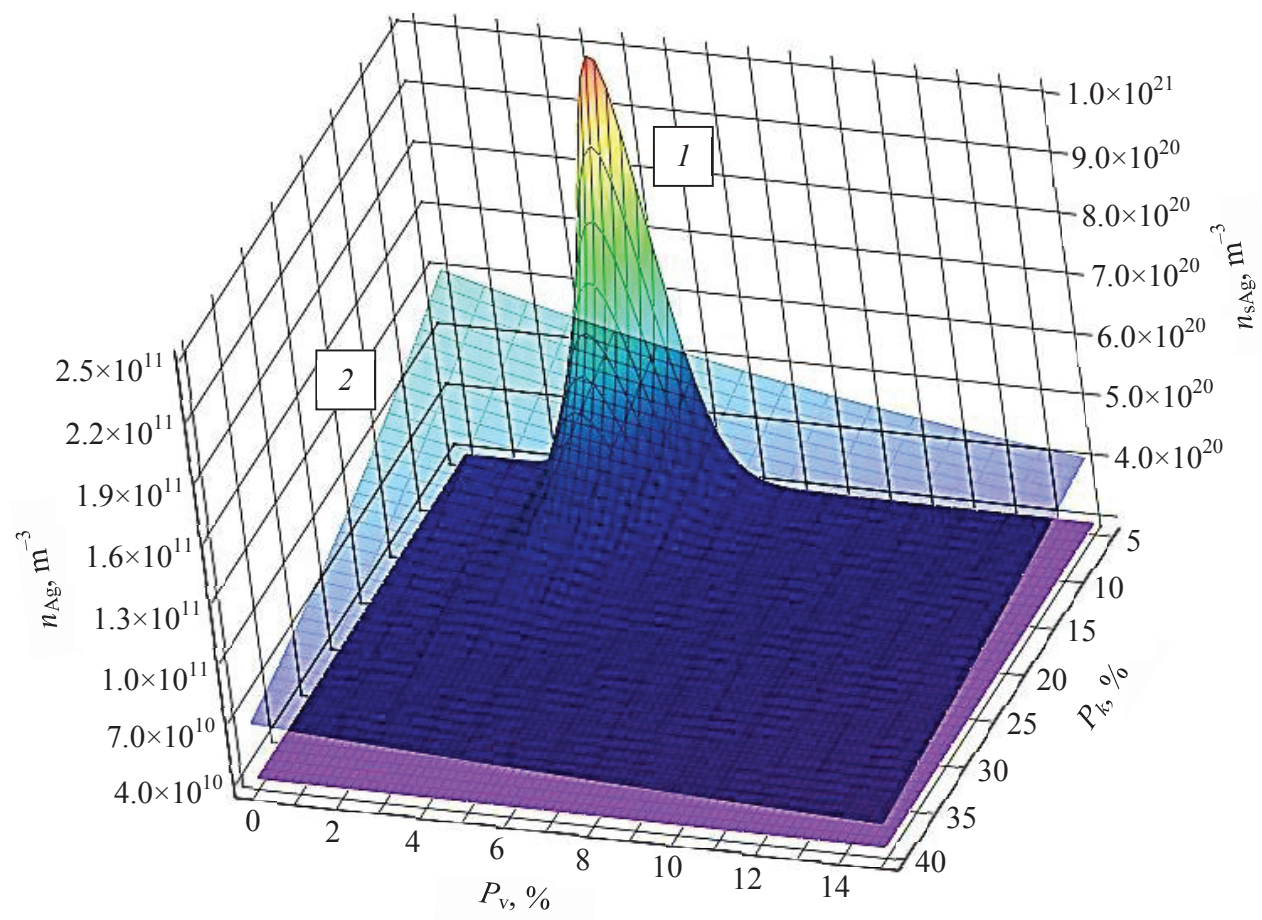

Рис. 2. Изменение концентраций поверхностных (1) и глубинных (2) атомов $\mathrm{Ag}$ в $\mathrm{MK} \mathrm{AgBr}$ в зависимости от $P_{k}$ и $P_{\mathrm{v}}$ при $T_{R}=298 \mathrm{~K}$

[Fig. 2. The changes in the concentrations of surface (1) and deep (2) Ag atoms in AgBr microcrystals as a function of $P_{k}$ and $P_{\mathrm{v}}$ at the $\left.T_{R}=298 \mathrm{~K}\right]$ 
$\sigma_{f} \approx 3.6 \cdot 10^{-9} \mathrm{~S} / \mathrm{m}$. Этот факт имеет экспериментальное подтверждение в [2] и объясняется низким экранирующим эффектом электрического поля за счет незначительного взаимовлияния кристалликов друг на друга благодаря их малой концентрации в фотоэмульсии и максимальному действию поля на каждый MK AgHal. Однако в этом случае, как известно, будет уменьшаться оптическая плотность изображений, несмотря на эффективность процесса электрополевой коалесценции как поверхностных, так и глубинных атомов Ag на каждом кристаллике. Поэтому с практической точки зрения целесообразно выбирать при синтезе электрочувствительных AgHal-фотоэмульсий компромиссный вариант между величинами $P_{k}$ и $P_{\mathrm{v}}$.

Из данных моделирования следует весьма обширный перечень параметров, вариацией которых возможно управление коалесценцией атомов Ag и, как следствие, ЭПЧ AgHal-фотоматериалов. С практической точки зрения интерес представляют следующие ситуации: а) когда для беспримесного MK AgBr малое изменение температуры в 6 К приводит к быстрому росту поверхностных атомов Ag при естественном влагосодержании желатины (обычно составляющем от 6 до 8 \% [11]), что эквивалентно увеличению ЭПЧ; б) достижение такого же эффекта путем замещения в MK AgHal 5 \% ионов $\mathrm{Br}^{-}$ионами I- при фиксированной $T_{R}=298 \mathrm{~K}$.

\section{ЭКСПЕРИМЕНТАЛЬНАЯ ЧАСТЬ}

Экспериментальная проверка результатов физико-математического моделирования осуществлялась как на промышленно выпускаемых

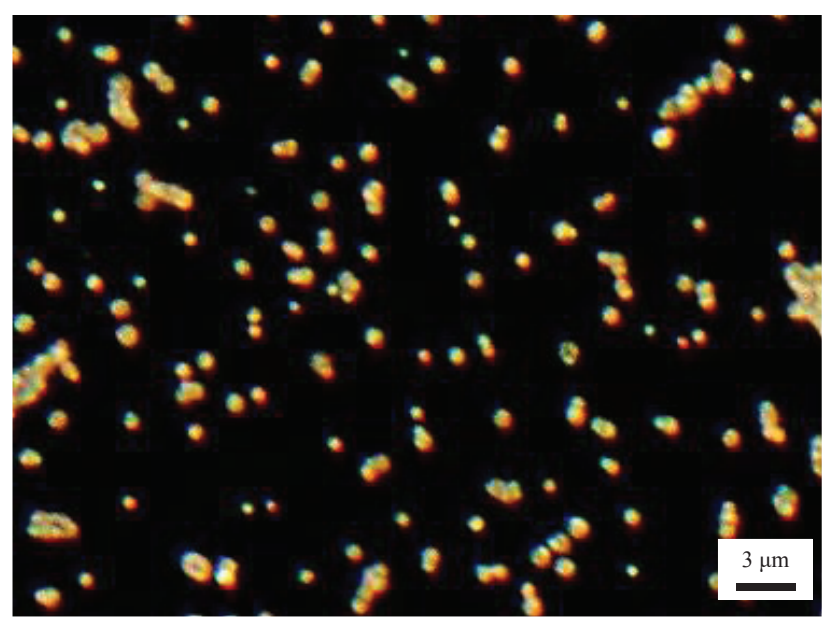

Рис. 3. Оптическая микрофотография синтезированных MK AgBr

[Fig. 3. Optical microphotograph of synthesized $\mathrm{AgBr}$ microcrystals]
AgHal-фотоматериалах, так и изготовленных путем полива на полимерную подложку специально синтезированных фотоэмульсий, содержащих МК сферической формы и средних размеров $10^{-6} \mathrm{~m}$, в которых доля анионных примесей $\mathrm{I}^{-}$от ионов $\mathrm{Br}^{-}$составляла 0,5 и 45 \% соответственно. На рис. 3 представлено оптико-микроскопическое изображение синтезированных МК на примере AgBr. B первую группу были отобраны фотопленки для медицинской рентгенографии «Retina», выпускаемые немецкой фирмой «FotoHandelsgesellschaft MBH», и «Agfa»- бельгийской фирмой «Agfa HealtheCare NV». Последний фотоматериал при стандартных термодинамических условиях обладает рекордно высокой ЭПЧ $[8,9]$, соответствуя $\Theta=0.22$ благодаря удачному балансу его $\sigma_{p}=8.7 \cdot 10^{-13} \mathrm{~S} / \mathrm{m}$ и $\sigma_{f}=2.0 \cdot 10^{-11} \mathrm{~S} / \mathrm{m}$, поэтому на полимерную подложку от него наносились синтезированные AgHal-фотоэмульсии. Все они подвергались сернисто-золотой сенсибилизации по технологии [19], а изготовленные на их основе фотопленки были отнесены ко второй группе, как удовлетворяющие выбранным значениям параметров модели. По величинам концентраций вводимых примесей анионов $\mathrm{I}^{-}$ в MK AgBr трем последним фотопленкам даны соответствующие названия: «ЭФМ-0», «ЭФМ-5» и «ЭФМ-45» (аббревиатура от слова «электрофотоматериал»).

Электрополевая экспозиция (ЭПЭ) всех образцов исследуемых фотоматериалов осуществлялась для каждого из них в пяти повторностях при неактиничном освещении (ЭПЭ фотопленки «Retina» осуществлялась через подложку от «Agfa» с целью локализации поляризационного заряда в фотоэмульсии) однократным импульсом напряжения длительностью $1 \mathrm{~s}$ разной полярности и амплитуды, которое прикладывалось между плоским и полусферическим электродами ЭТГ-ячейки, описанной в [9]. Эксперименты проводились при контролируемой температуре, поддерживаемой на уровне $(298.0 \pm 0.5) \mathrm{K}$, включая и условия химико-фотографической обработки фотоматериалов. (Для восстановления $\mathrm{Ag}$-центров скрытого ЭПИ до видимого использовался фенидон-гидрохиноновый проявитель, состав которого приведен в табл. 3). Аналогично описанным проводились эксперименты по термовлиянию на формирование ЭПИ, но с использованием холодильной камеры, в которой размещалась ЭТГ-ячейка с исследуемыми фотоматериалами. В этом варианте эксперимента выбиралась расчетная температура $(292.0 \pm 0.5) \mathrm{K}$, при которой наиболее высока эффективность 
Таблица 3. Состав раствора восстановителя Ag (проявителя)

[Table 3. The composition of the Ag reducing agent (developer)]

\begin{tabular}{|l|c|}
\hline \multicolumn{1}{|c|}{$\begin{array}{c}\text { Химический компонент } \\
\text { [Chemical component] }\end{array}$} & $\begin{array}{c}\text { * Macca в pacтворе, g } \\
\text { [* Weight in solution, g] }\end{array}$ \\
\hline Метилфенидон [Methylphenidone] & 0.8 \\
\hline Гидрохинон [Hуdroquinone] & 10 \\
\hline Натрий сернистокислый (безводный) & 60 \\
[Sodium sulfite (anhydrous)] & 50 \\
\hline Натрий углекислый [Sodium carbonate] или [or] & 65 \\
Калий углекислый [Potassium carbonate] & 3 \\
\hline Калий бромистый [Potassium bromide] & 0.1 \\
\hline ** Бензотриазол [Benzotriazole] & 0.007 \\
\hline ** 1-Фенил-5-меркаптотетразол [1-Phenyl-5-mercaptotetrazole] & \\
\hline
\end{tabular}

* Весовые значения компонент приведены на 1 литр дистиллированной воды

[*The weight values of the components are given in 1 liter of distilled water]

** Использовались этанольно-спиртовые растворы этих соединений, которые вводились в общий раствор при его приготовлении

[ ${ }^{* * *}$ Ethanol-alcohol solutions of these compounds were used, which were introduced into the total solution during its preparation]

коалесцентно-электрополевого процесса для поверхностных атомов Ag.

Полученные на фотопленках ЭПИ полусферического электрода (рис. 4) микро-денситометрировались по модернизированной методике [1]. Оценка оптической плотности ЭПИ $(D)$ осуществлялась по разнице обратных логарифмов интенсивностей световых потоков, прошедших через изображение $I_{s}$ и участок фотоматериала, не содержащего ЭПИ (оптическая вуаль) $I_{v}: D=\left(\lg I_{s}\right)^{-1}-\left(\lg I_{v}\right)^{-1}$. Кроме названного параметра определялись геометрические размеры изображений - диаметр их колец $L$. Для вы-

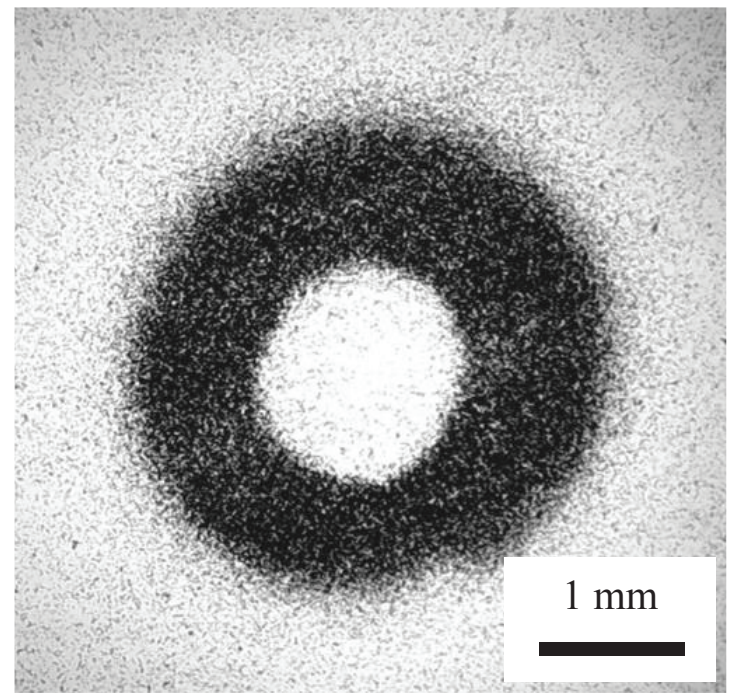

явления различий между опытными и контрольными образцами проводилась оценка «эффекта влияния» исследуемого фактора воздействия на оптическую плотность ЭПИ $\xi$ и их размеры $\chi$ по формулам: $\xi=\left(D^{\prime}-D_{0}\right) / D_{0}$ и $\chi=\left(L^{\prime}-L_{0}\right) / L_{0}$, где $D_{0}$ и $D^{\prime}$ - исходная оптическая плотность ЭПИ и измеренная при исследуемом факторе воздействия, а $L_{0}$ и $L^{\prime}$ - соответственно размеры ЭПИ, измеренные при тех же условиях. Результаты проведенных экспериментов представлены в табл. 4 и табл. 5 с погрешностью значений параметров, не превышающей $1.2 \%$.

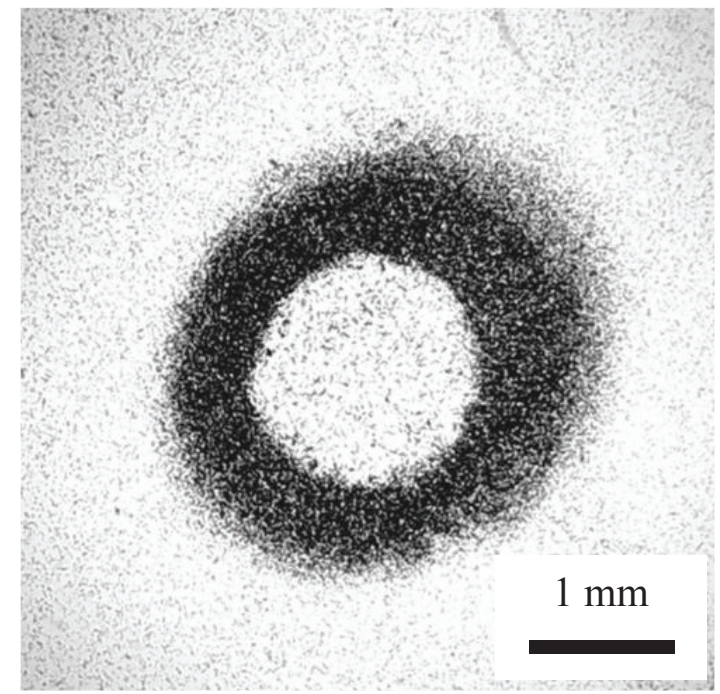

Рис. 4. Негативные ЭПИ полусферического электрода на фотопленке «ЭФМ-5» при положительной (слева) и отрицательной (справа) полярности напряжения в $(1.00 \pm 0.01) \mathrm{kV}$

[Fig. 4. EFI-negative of hemispherical electrode on photo-film «EPhM-5» with positive (left) and negative (right) polarity of voltage in $(1.00 \pm 0.01) \mathrm{kV}]$ 
Таблица 4. Результаты термовлияния на формирование ЭПИ при напряжении $(1.00 \pm 0.01) \mathrm{kV}$

[Table 4. The results of thermal influence on the formation of electric field images at a voltage $(1.00 \pm 0.01) \mathrm{kV}]$

\begin{tabular}{|c|c|c|c|c|c|c|c|}
\hline \multirow{2}{*}{$\begin{array}{c}\text { Фото-материал } \\
\text { [Photographic } \\
\text { material] }\end{array}$} & \multirow{2}{*}{$\begin{array}{l}\text { Поляр- } \\
\text { ность } \\
\text { [Polarity] }\end{array}$} & \multicolumn{2}{|c|}{$\begin{array}{c}\text { Оптическая } \\
\text { плотность ЭПИ } \\
\text { [Optical density of EFI] }\end{array}$} & \multicolumn{2}{|c|}{$\begin{array}{l}\text { Размер ЭПИ, } 10^{-3} \mathrm{~m} \\
\left.\text { [EFI size, } 10^{-3} \mathrm{~m}\right]\end{array}$} & \multicolumn{2}{|c|}{$\begin{array}{l}\text { «Эффект влияния» } \\
\text { [«Effect of influence»] }\end{array}$} \\
\hline & & $D_{0}$ & $D^{\prime}$ & $L_{0}$ & $L^{\prime}$ & $\xi$ & $\chi$ \\
\hline \multirow{2}{*}{ «Agfa» } & + & 2.48 & 4.69 & 1.73 & 2.77 & 0.89 & 0.60 \\
\hline & - & 0.84 & 3.15 & 0.65 & 1.03 & 2.75 & 0.58 \\
\hline \multirow{2}{*}{ «RETINA» } & + & 2.21 & 3.07 & 1.83 & 2.06 & 0.39 & 0.13 \\
\hline & - & 2.42 & 3.00 & 1.15 & 1.25 & 0.24 & 0.09 \\
\hline \multirow{2}{*}{$\begin{array}{l}\text { «ЭФM-0» } \\
{[\text { EPhM-0] }}\end{array}$} & + & 0.32 & 1.68 & 0.85 & 1.02 & 4.25 & 0.20 \\
\hline & - & 0.30 & 2.40 & 0.55 & 0.61 & 7.00 & 0.11 \\
\hline
\end{tabular}

Таблица 5. Результаты влияния анионных примесей в MK AgHal на формирование ЭПИ при $T_{R}=298 \mathrm{~K}$

[Table 5. The results of an anion-impurity effect in AgHal microcrystal on the formation of electric field images at $\left.T_{R}=298 \mathrm{~K}\right]$

\begin{tabular}{|c|c|c|c|c|c|}
\hline \multirow{2}{*}{$\begin{array}{c}\text { Фотоматериал } \\
\text { [Photographic } \\
\text { material] }\end{array}$} & \multirow{2}{*}{$\begin{array}{l}\text { Полярность } \\
\text { [Polarity] }\end{array}$} & \multirow{2}{*}{$\begin{array}{c}\text { Оптическая } \\
\text { плотность ЭПИ } \\
\text { [Optical density of EFI] }\end{array}$} & \multirow{2}{*}{$\begin{array}{l}\text { Размер ЭПИ, } 10^{-3} \mathrm{~m} \\
\left.\text { [EFI size, } 10^{-3} \mathrm{~m}\right]\end{array}$} & \multicolumn{2}{|c|}{$\begin{array}{l}\text { «Эффект влияния» } \\
\text { [«Effect of influence»]* }\end{array}$} \\
\hline & & & & $\xi$ & $\chi$ \\
\hline \multicolumn{6}{|c|}{$\begin{array}{c}\text { Амплитуда импульса напряжения }(1.00 \pm 0.01) \mathrm{kV} \\
\text { [Amplitude of the voltage pulse }(1.00 \pm 0.01) \mathrm{kV}]\end{array}$} \\
\hline \multirow{2}{*}{$\begin{array}{l}\text { «ЭФM-0» } \\
{[\mathrm{EPhM}-0]}\end{array}$} & + & \begin{tabular}{|c|}
0.32 \\
\end{tabular} & 0.85 & - & - \\
\hline & - & 0.30 & 0.55 & - & - \\
\hline \multirow{2}{*}{$\begin{array}{l}\text { «ЭФМ-5» } \\
{[\text { EPhM-5] }}\end{array}$} & + & 0.61 & 0.97 & 0.91 & 0.14 \\
\hline & - & 0.45 & 0.75 & 0.50 & 0.36 \\
\hline \multirow{2}{*}{$\begin{array}{l}\text { «ЭФM-45» } \\
{[\mathrm{EPhM}-45]}\end{array}$} & + & 0.13 & 1.17 & -0.59 & 0.38 \\
\hline & - & 0.02 & 0.67 & -0.93 & 0.22 \\
\hline \multicolumn{6}{|c|}{$\begin{array}{c}\text { Амплитуда импульса напряжения }(1.50 \pm 0.01) \mathrm{kV} \\
\text { [Amplitude of the voltage pulse }(1.50 \pm 0.01) \mathrm{kV}]\end{array}$} \\
\hline \multirow{2}{*}{$\begin{array}{l}\text { «ЭФМ-0» } \\
{[\mathrm{EPhM}-0]}\end{array}$} & + & \begin{tabular}{|c|}
0.56 \\
\end{tabular} & 1.80 & - & - \\
\hline & - & 0.43 & 1.05 & - & - \\
\hline \multirow{2}{*}{$\begin{array}{l}\text { «ЭФМ-5» } \\
{[\mathrm{EPhM}-5]}\end{array}$} & + & 2.97 & 2.00 & 4.30 & 0.11 \\
\hline & - & 1.63 & 1.50 & 2.79 & 0.43 \\
\hline \multirow{2}{*}{$\begin{array}{l}\text { «ЭФМ-45» } \\
{[\mathrm{EPhM}-45]}\end{array}$} & + & 0.22 & 2.03 & -0.61 & 0.13 \\
\hline & - & 0.18 & 1.20 & -0.58 & 0.14 \\
\hline
\end{tabular}

* «Эффект влияния» оценивался относительно параметров ЭПИ, сформированных на фотопленке «ЭФМ-0» при заданном напряжении

[* The «Effect of influence» was evaluated with respect to the EFI parameters formed on the «EPhM-0» film at a given voltage]

\section{РЕЗУЛЬТАТЫ И ИХ ОБСУЖДЕНИЕ}

Как видно из полученных результатов эксперимента, они повторяют основные закономерности коалесцентно-электрополевого процесса на атомах Ag в MK AgHal, установленные при физико-математическом моделировании. Особенно четко это выражено на фотопленках со специально синтезированными фотоэмульсиями. Так, эффект термовлияния на $D$ ЭПИ для фотопленки
«ЭФМ-0» в 1.5 и более раз превосходит этот параметр для промышленных фотопленок «Agfa» и «Retina» (табл. 4). Данный факт явно указывает на наличие в последних добавок или покрытий, увеличивающих общую электропроводность рентгеновских фотоматериалов, обычно вводимых или наносимых для устранения электростатического эффекта во время их эксплуатации. С точки зрения электрополевой фотографии такие 
добавки уменьшают ЭПЧ фотоматериалов, поэтому в синтезированные эмульсии они не вводились. Тем не менее, на всех фотопленках обнаружен положительный эффект термовлияния, как по $D$ ЭПИ, так и их размерам при обеих полярностях прикладываемого напряжения.

Примесь анионов $\mathrm{I}^{-}$в MK AgBr также изменяет исходную $D$ ЭПИ и соответственно, ЭПЧ фотоматериалов (табл. 5). Однако, как и предсказано моделью, этот фактор эффективен лишь при небольших концентрациях вводимого аниона. При однокиловольтных импульсах положительной полярности $D$ ЭПИ электрода на фотопленке «ЭФМ-5» почти в 2 раза превышает этот параметр для пленки «ЭФМ-0» и в 1.5 раза - для импульсов напряжения той же амплитуды отрицательной полярности. Увеличение экспонирующего напряжения до $1.5 \mathrm{kV}$ увеличивает исходную плотность и размеры ЭПИ, но не меняет знака эффектов ни по $\xi$, ни по $\chi$ для фотоэмульсионных МК с анионными примесями. В случае импульсов положительной полярности $D$ ЭПИ электрода между пленками «ЭФМ-5» и «ЭФМ-0» различается в 5.3 раза, а при отрицательной полярности - в 3.8 раза. Для размеров ЭПИ эти различия меньше и соответственно составляют 1.1 и 1.4 раза при напряжении $1.5 \mathrm{kV}$.

Противоположный, отрицательный эффект анионно-примесного влияния $\mathrm{I}^{-}$наблюдается для $\xi$ между фотопленками «ЭФМ-45» и «ЭФМ-0» при разнополярном напряжении и разной амплитуде. При ЭПЭ импульсами напряжения $1 \mathrm{kV}$ положительной полярности на пленке «ЭФМ-45» обнаруживается $D$ ЭПИ электрода почти в 2.5 раза меньше того же параметра на пленке «ЭФМ-0», а при отрицательной - в 15 раз. При амплитуде $1.5 \mathrm{kV}$ соответствующее снижение составляет 2.5 и 2.4 раза для разнополярных импульсов. Согласно разработанной модели и данных [16], наблюдаемую закономерность можно объяснить следующими соображениями. При замещении в $\mathrm{MK} \mathrm{AgBr}$ ионов $\mathrm{Br}^{-}$ионами $\mathrm{I}^{-}$ , после 42 \% наблюдается образование двух фаз фотоэмульсионных МК, состоящих из кристалликов AgHal смешанного состава (содержащих в кристаллической решетке ионы $\mathrm{Br}^{-}$и I- определенного соотношения) и отдельно AgI. Такое фазообразование изменяет электрофизические свойства фотоэмульсии даже при постоянных величинах $P_{k}$ и $P_{\mathrm{v}}$. Причем, наибольшие изменения отражаются именно на ее электропроводности. Так, например, замещение в $\mathrm{MK} \mathrm{AgBr}$ ионов I- концентрацией от 0 до $30 \%$ уменьшает $\varepsilon_{f}$ всего на 0.38 единиц, а $\sigma_{f}$ при этом падает в
2.3 раза. В свою очередь такие изменения существенно влияют на время ионной релаксации фотоэмульсии и как следствие - на распределение электрических полей в объеме МК и на их поверхности. Понятно, что наличие в фотоэмульсии новой фазы из AgI, которой соответствует фотопленка «ЭФМ-45», лишь усилит электрополевое перераспределение между МК за счет существенных различий электрофизических параметров между AgI и $\mathrm{AgBr}$ [16]. Такое перераспределение полей эквивалентно уменьшению ЭПЭ и соответственно ЭПЧ фотоматериала.

В связи с тем, что кинетическая модель [10] относится к микроскопическим и не позволяет предсказывать изменений размеров ЭПИ под действием каких-либо факторов, представляются интересными экспериментальные результаты по оценке параметра $\chi$. Между ним и $\xi$ имеется определенная, хотя и неоднозначно интерпретируемая корреляция. Так, с изменением $T$ при импульсах отрицательной полярности эффект $\xi$ для фотопленок «Agfa» и «ЭФМ-0» оказывается больше, чем при положительной полярности, а для параметра $\chi$ наблюдается противоположная закономерность для всех фотопленок. Лишь у фотопленки «Retina» тенденция изменения величин $\chi$ совпадает с $\xi$ при разнополярном напряжении, что, по-видимому, связано с ее ЭПЭ через подложку от фотопленки «Agfa» (в противном случае ЭПИ на «Retina» вообще не формируются). Сказанное справедливо и для фотоэмульсионных МК с анионными примесями IДля пленок «ЭФМ-5» и «ЭФМ-45» при напряжении 1 и $1.5 \mathrm{kV}$ обеих полярностей тенденция изменения величин эффектов $\chi$ и $\xi$ носит между собой противоположный характер.

Особого внимания заслуживает знак эффекта $\chi$ для фотопленки «ЭФМ-45». В отличие от параметра $\xi$ он имеет положительный знак при обеих полярностях напряжения разной амплитуды, что на первый взгляд не является очевидным фактом. Было бы естественно ожидать уменьшения размеров изображений с падением их $D$, ибо с наблюдаемым для других фотоматериалов ростом $D$ ЭПИ также растут и их размеры. Рост размеров изображений относительно их исходных значений означает вовлеченность в коалесцентно-электрополевой процесс большего количества фотоэмульсионных MK AgHal, распределенных на единице площади фотоматериала. В случае фотопленки «ЭФМ-45», как уже отмечалось, имеется две фазы разнообъемных долей МК в фотоэмульсии. Очевидно, что с большей вероятностью в коалесцентно-электрополевой 
процесс будет вовлечена фаза из MK AgHal смешанного состава, имеющая большую объемную долю в фотоэмульсии по сравнению с фазой МК из чистого AgI. Судя по данным $\chi$ и результатам моделирования можно предположить, что в сравнении с MK AgI наибольшей ЭПЧ обладают смешанные кристаллики AgHal, но меньшей, чем, например, у чистого $\mathrm{AgBr}$, поэтому на фотопленке «ЭФМ-45» и реализуются эффекты $\xi$ и $\chi$ с противоположными знаками.

\section{ЗАКЛЮЧЕНИЕ И ВЫВОДЫ}

Чуть меньше четверти века назад AgHal-фотография уступила свое лидерство цифровой. Однако, по мнению ведущих специалистов [20], эта уступка относится лишь к оптической части электромагнитного спектра, давшая толчок развитию физико-химии наночастиц [21]. Ядернофизический эксперимент, физика космических лучей, голография, радио- и рентгенография пока не торопятся к отказу от использования уникальных свойств MK AgHal, чувствительных не только к действию фотонного излучения. Как показано в более ранних работах и настоящей, они проявляют высокую ЭПЧ (порой превосходящую свето- или рентгеночувствительность того же материала) и, в отличие от цифровых приемников оптической информации, допускают ее регистрацию непосредственным размещением в электрических полях большой напряженности. Это дало нам основание назвать данный вид фотографии «электрополевая» [9]. Независимо и одновременно с нашими работами по обнаружению магнитоэлектротопографического эффекта на AgHal-материалах [22], группой исследователей Воронежского университета был заложен фундамент для зарождения еще одной разновидности фотографии [23] - магнитополевой, ждущей своего научного обоснования и применения. Таким образом, научная фотография на основе MK AgHal далека от завершения и исчерпания, примером чего является ЭТГ-эффект, многие теоретические и прикладные аспекты которого удалось обосновать лишь в XXI веке. Этому существенно способствовала разработка кинетической модели электрополевой коалесценции атомов Ag в МК его галогенидов [10], а также экспериментальные результаты ее апробации, полученные ранее и в настоящей работе. Из обобщения последних можно сделать следующие основные выводы:

1. С помощью кинетической модели электрополевой коалесценции атомов Ag в МК его гало- генидов теоретически показана возможность управления этим процессом и, как следствие, ЭПЧ AgHal-фотоматериалов путем вариации многих макро- и микроскопических параметров, например таких, как температура, влагосодержание фотоэмульсии, объемная доля содержащихся в ней MK AgHal, а также концентрация замещающих в МК ионов $\mathrm{Br}^{-}$ионами $\mathrm{I}^{-}$.

2. Теоретически установлено, что эффективность коалесцентно-электрополевого процесса на атомах Ag может достигаться при уменьшении температуры (относительной стандартной в $298 \mathrm{~K})$, либо замещением в МК 5 \% ионов $\mathrm{Br}^{-}$ ионами $\mathrm{I}^{-}$, а также вариацией влагосодержания фотоэмульсии в диапазоне от 3 до 8 \% или концентрации содержащихся в ней MK AgHal от 5 до $30 \%$.

3. Для практически интересных ситуаций на промышленно выпускаемых фотопленках «Agfa» и «Retina», а также специально изготовленных с фотоэмульсионными MK AgHal, в которых доля анионных примесей $\mathrm{I}^{-}$от ионов $\mathrm{Br}^{-}$ составляет 0, 5 и $45 \%$, экспериментально показана корреляция изменения $D$ ЭПИ с результатами физико-математического моделирования. При этом установлено, что наибольшая эффективность коалесцентно-электрополевого процесса и соответственно ЭПЧ достигается при криотермическом воздействии для фотопленки «ЭФМ-0», содержащей беспримесные $\mathrm{MK} \mathrm{AgBr}$, a при $T_{R}=298 \mathrm{~K}$ - для фотопленки «ЭФМ-5» с $5 \%$ содержанием ионов $\mathrm{I}^{-}$в МК.

4. При разнополярных импульсах напряжения между $D$ ЭПИ и их размерами выявлена определенная корреляция. Независимо от термического или анионно-примесного фактора воздействия при импульсе какой-то одной полярности, параметр $\xi$ оказывается меньше $\chi$ и, наоборот, при импульсе противоположной полярности. Исключением из этого правила оказалась фотопленка «Retina» по причине, вероятно связанной с ее ЭПЭ через подложку от «Agfa». У фотопленки «ЭФМ-45» эффекты $\xi$ и $\chi$ хотя и сохраняют описанную корреляцию, но имеют противоположные знаки, что очевидно связано с наличием двух фаз МК в фотоэмульсии, состоящих из чистого AgI и AgHal смешанного состава галогенов.

\section{КОНФЛИКТ ИНТЕРЕСОВ}

Авторы сообщают об отсутствии конфликтов интересов, повлиявших на результаты исследования или их интерпретацию. 


\section{СПИСОК ЛИТЕРАТУРЫ}

1. Кравцов А. Е., Резников М. А., Пипа В. И., Фок М. В. О природе электрочувствительности фотографических эмульсионных слоев // Журнал научной и прикладной фото- и кинематографии, 1977, т. 22(3), с. 186-195.

2. Кравцов А. Е., Пипа В. А., Резников М. А., Фок М. В. Электротопографический эффект в фотоэмульсиях и его применения // Труды ФИАН, 1981, т. 129 , с. 13-65.

3. Fok M. V., Reznikow M. A., Kravtsov A. E. Coalescence of silver atoms on the surface of a silverhalide crystal in the electric field // J. Signal AM, 1982, vol. 10(5), pp. 303-309.

4. Галашин Е. А. Термодинамическая теория фотографического процесса // Журнал научной $u$ прикладной фото- и кинематографии, 1968, т. 13(3), c. 203-210.

5. Senchenkov E. P., Galashin E. A., Fedorov Yu V., Chibisov K. V. Electron microscope studies of photographic process and nature of photographic sensitivity // J. Signal AM, 1982, vol. 10(1), pp. 3-15

6. Резников М. А., Фок М. В. Устойчивость серебряных кластеров на поверхности галогенида серебра // Успехи научной фотографии, 1986, т. 24, c. $143-157$.

7. Гречко Г. М. Современные достижения космонавтики. Сборник статей. Сер. Космонавтика, астрономия. М.: Знание, 1983(12), с. 3-32.

8. Шишканов О. Н., Бойченко А. П. О высокой электрополевой чувствительности рентгеновксой фотопленки «Agfa» «Bопросы образования и науки: теоретический и методический аспекты». Сборник научных трудов Международной научно-практической конференции, 31 мая 2014 г., Тамбов, 2014, c. $170-171$.

9. Шишканов О. Н., Бойченко А. П., Яковенко Н. А. Химико-фотографические исследования процессов электрополевой коалесценции атомов серебра в микрокристаллах его галогенидов // Экологический вестник научных центров Черноморского экономического сотрудничества (ЧЭС), 2015(2), c. $89-100$.

10.Шишканов О. Н., Бойченко А. П. Кинетическая модель коалесценции атомов серебра в микрокристаллах его галогенидов под действием электрического поля // Фундаментальные исследования, 2014(8-3), с. 607-613.URL:http://fundamentalresearch.ru/ru/article/view?id=34602 (дата обращения: 06.05.2019).

11. Гущин Е. М., Лебедев А. Н., Сомов С. В. О диэлектрических свойствах галогенсеребряных фотоэмульсий // Журнал научной и прикладной фото- и кинематографии, 1986, т. 31(2), с. 95-99.

12. Ханефт А. В., Крашенинин В. И. Кинетика поляризации и эффект термогенерации дефектов Френкеля в галогенидах серебра // Журнал научной и прикладной фото- и кинематографии, 1998, т. 43(1), с. 34-43.

13. Молоцкий М. И., Латышев А. Н. К устойчивости агрегатов F-центров в бромиде серебра // Журнал научной и прикладной фото- и кинематографии, 1969, т. 14(5), с. 380-382.

14. Латышев А. Н., Леонова Л. Ю., Саввин Н. И. Химико-физическая модель сернисто-серебряных центров светочувствительности // Журнал научной и прикладной фото- и кинематографии, 1995, т. 40(6), c. 18-22.

15. Иноуе К., Китахара А., Косеки С. и др. Капиллярная химия: Пер. с японского. М.: Мир, 1983. $272 \mathrm{c.}$

16. Мейкляр П. В. Физические процессы при образовании скрытого фотографического изображения. М.: Наука, 1972. 400 с.

17. Шишканов О. Н., Овеченко Д. С., Бойченко А. П. Температурная зависимость диэлектрической проницаемости галогенсеребряных фотоэмульсий с различным влагосодержанием. «Приоритетные научные направления: от теории $\mathrm{k}$ практике». Сборник материалов Х Международной научно-практической конференции, 10 апреля 2014 г., Новосибирск, 2014, с. 102-106.

18. Латышев А. Н., Молоцкий М. И., Чибисов К. В. Образование серебряных частиц вблизи мест выхода дислокации на поверхность бромида серебра // Журнал научной и прикладной фото- $u$ кинематографии, 1976, т. 21(3), с. 161-165.

19. Сидоров Ю. Д., Ли Н. И., Крестникова Е. Д., Сидорова Т. В. Способ изготовления йодбромсеребряной фотографической эмульсии. Авторское свидетельство № 883845 РФ. 1981, бюл. изобр. № 43.

20. Редько А. В. Основы фотографических процессов. СПб.: Лань, 1999, 512 с.

21. Латышев А. Н., Овчинников О. В. От научной фотографии к физике наночастиц // Вестник ВГУ, сер. физика, математика, 2013(1), с. 63-93.

22. Бойченко А. П. Изучение влияния магнитного поля высокой напряженности на электротопографический эффект. «Опто-, наноэлектроника, нанотехнологии и микросистемы». Труды VII Международной конференции, Ульяновск, 2005. С. 23.

23. Волошина Т. В., Дронов М. А., Ефимова М. А., Латышев А. Н., Левин М. Н., Москинов В. А. Влияние магнитного поля на свойства фотоматериалов // Химия высоких энергий, 2005, т. 39(3), с. 213-217. 
UDC 535.662.2+778.68

DOI: https://doi.org/10.17308/kcmf.2019.21/769

Received 03.04.2019

Accepted 15.05.2019

\title{
THE EFFECT OF THERMAL AND ANION-IMPURITY FACTORS ON THE ELECTRO-FIELD COALESCENCE OF SILVER ATOMS IN ITS HALIDE MICROCRYSTALS
}

\author{
(c) 2019 O. N. Shishkanov, A. P. Boychenko \\ Kuban State University \\ 149, Stavropolskaya str., 350040 Krasnodar, Russian Federation
}

\begin{abstract}
Purpose. The discovery, in 1970, of the "electrotopographic" effect on halogen-silver photographic materials contributed to the appearance of the photograph of a new kind, later called "electro-field photography". The development of the physical and mathematical model of the kinetics of the electro-field coalescence of silver atoms in its halide microcrystals in 2014 allowed substantiating the previously existing theoretical and applied aspects of the process and predicting the conditions for controlling it. This paper is dedicated to the experimental verification of the influence of the cryothermic and anion-impurity factors on this process in the form of iodine ions, replacing bromine ions in silver halide microcrystals.

Methods and methodology. The study of the thermal influence was carried out on commercially available films for radiographs "Agfa" and "Retina", as well as on specially made photographic films with $\mathrm{AgBr}$ microcrystals with a decreased temperature compared to the standard $298 \mathrm{~K}$. The study of the effect of anion-impurity factors was tested on specially manufactured photographic films with mixed AgHal microcrystals with different amounts of iodine ions and bromine ions.

Results and conclusions. Using both commercially produced films for radiographs and specially made films showed the gain in the coalescence process of silver atoms affected by the electric field with a decrease in temperature from 298 to $292 \mathrm{~K}$, or a $5 \%$ substitution of bromine ions to iodine ions in $\mathrm{AgBr}$ crystals at $298 \mathrm{~K}$. A certain correlation was found between the optical density of the electro-field images and their dimensions with voltage impulses of varying polarity. Regardless of the thermal or anion-impurity factor, the parameter $\xi$ appears to be smaller than $\chi$ with the impulse of the same polarity, and vice versa with the impulse of the opposite polarity. An exception to this rule was the "Retina" film. This can be due to its exposure to the electric field through the base of the "Agfa" film. The effects $\xi$ and $\chi$ for the "EPhM-45" film, while retaining the described correlation, have opposing qualities, which is obviously due to the presence of two phases of microcrystals in a photo-emulsion consisting of a pure AgI and AgHal with a mixed composition of halogens.
\end{abstract}

Keywords: electric field image, electric field sensitivity, silver halides, coalescence of silver atoms and clusters, thermal influence, anions impurities.

\section{CONFLICT OF INTEREST}

Authors report no conflicts of interest affecting research results or their interpretation.

\section{REFERENCES}

1. Kravcov A. E., Reznikov M. A., Pipa V.I., Fok M. V. Zhurnal nauchnoj i prikladnoj foto- $i$ kinematografi, 1977, v. 22(3), pp. 186-195. (in Russ.)

2. Kravcov A. E., Reznikov M. A., Pipa V. I., Fok M. V. Elektrotopograficheskiy effekt $v$ fotoemul'siyakh i ego

$\triangle$ Boychenko Aleksandr P., e-mail: bojchenco@yandex.ru primeneniya [Electrophotographic effect in photoemulsions and its applications]. Trudy FIAN, 1981(129), pp. 13-65. (in Russ.)

3. Fok M. V., Reznikow M. A., Kravtsov A. E. J. Signal AM, 1982, v. 10(5), pp. 303-309.

4. Galashin E. A. Zhurnal nauchnoj i prikladnoj fotoi kinematografii, 1968, v. 13(3), pp. 203-210. (in Russ.)

5. Senchenkov E. P., Galashin E. A., Fedorov Yu V., Chibisov K. V. Electron microscope studies of photographic process and nature of photographic sensitivity. J. Signal AM, 1982, vol. 10(1), pp. 3-15. 
6. Reznikov M. A., Fok M. V. Uspehi nauchnoj fotografii, 1986, v. 24, pp. 143-157. (in Russ.)

7. Grechko G. M. Sovremennye dostizhenija kosmonavtiki [Modern achievements of astronautics]. Moscow, Znanie Publ., 1983(12), pp. 3-32. (in Russ.)

8. Shishkanov O. N., Boychenko A. P. «Issues of Education and Science: Theoretical and Methodological Aspects». Proceedings of the International scientificpractical Conference, May 31, 2014, Tambov, 2014, pp. 170-171. (in Russ.)

9. Shishkanov O. N., Boychenko A. P., YAkovenko N. A. Khimiko-fotograficheskie issledovaniya protsessov elektropolevoy koalestsentsii atomov serebra v mikrokristallakh ego galogenidov [Chemicalphotographic studies of the processes of electropole coalescence of silver atoms in the microcrystals of its halides]. Ehkologicheskij vestnik nauchnyh centrov CHernomorskogo ehkonomicheskogo sotrudnichestva (CHEHS), 2015(2), pp. 89-100. (in Russ.)

10. Shishkanov O. N., Boychenko A. P. Kinetic model coalescence atoms silver in microcrystals halogens under action an electrical field. Fundamental research, 2014(8), pt. 3, pp. 607-613. URL: http://fundamental-research.ru/ru/article/view?id=34602

11. Gushchin E. M., Lebedev A. N., Somov S. V. Zhurnal nauchnoj i prikladnoj foto- $i$ kinematografii, 1986, vol. 31(2), pp. 95-99. (in Russ.)

12. Haneft A. V., Krasheninin V. I. Kinetika polyarizatsii i effekt termogeneratsii defektov Frenkelya v galogenidakh serebra [Kinetics of polarization and the effect of thermogeneration of Frenkel defects in silver halides]. Zhurnal nauchnoj i prikladnoj foto- $i$ kinematografii, 1998, v. 43(1), pp. 34-43. (in Russ.)

13. Molockij M. I., Latyshev A. N. K ustoychivosti agregatov F-tsentrov $\mathrm{v}$ bromide serebra [To the stability of aggregates of F centers in silver bromide]. Zhurnal nauchnoj i prikladnoj foto- i kinematografii, 1969, v. 14(5), pp. 380-382. (in Russ.)

14. Latyshev A. N., Leonova L. Ju., Savvin N. I. Khimiko-fizicheskaya model' sernisto-serebryanykh tsentrov svetochuvstvitel'nosti [Chemical-physical model of sulfur silver sensitivity centers]. Zhurnal nauchnoj i prikladnoj foto- $i$ kinematografii, 1995, vol. 40(6), pp. 18-22. (in Russ.)

15. Inoue K., Kitahara A., Koseki S., at al. Kapilljarnaja himija: Per. s japonskogo. [Capillary chemistry: Trans. from japanese]. Moscow, Nauka Publ., 1983, 272 p. (in Russ.)

16. Mejklyar P. V. Fizicheskie processy pri obrazovanii skrytogo fotograficheskogo izobrazheniya [Physical processes in the formation of a hidden photographic image]. Moscow, Nauka Publ., 1972, 400 p. (in Russ.)

17. Shishkanov O. N., Ovechenko D. S., Boychenko A. P. «Priority research areas: from theory to practice». Proceedings of the $\mathrm{X}$ International scientific-practical Conference, April 10, 2014, Novosibirsk, 2014, pp. 102106. (in Russ.)

18. Latyshev A. N., Molockij M. I., Chibisov K. V. Obrazovanie serebryanykh chastits vblizi mest vykhoda dislokatsii na poverkhnost' bromida serebra [Formation of silver particles near the sites of dislocation on the surface of silver bromide]. Zhurnal nauchnoj i prikladnoj foto- i kinematografii, 1976, vol. 21(3), pp. 161-165. (in Russ.)

19. Sidorov Yu. D., Li N. I., Krestnikova E. D., Sidorova T. V. Author's certificate of the USSR no. 883845 , 1981. (in Russ.)

20. Red'ko A. V. Osnovy fotograficheskih processov [Foundation of photographic processes]. St. Petersburg, Lan’ Publ., 1999, 512 p. (in Russ.)

21. Latyshev A. N., Ovchinnikov O. V. From photographic science to nanoparticle physics. Vestnik $V G U$, ser. fizika, matematika [Proceedings of Voronezh State University. Series: Physics. Mathematics], 2013(1), pp. 63-93. (in Russ.)

22. Boychenko A. P. «Opto-, nanoelectronics, nanotechnologies and microsystems». Proceedings of the VII International Conference, Ulyanovsk, 2005, p. 23. (in Russ.)

23. Voloshina T. V., Dronov M. A., Efimova M. A., Latyshev A. N., Levin M. N., Moskinov V. A. Effect of magnetic field on the properties of photographic materials. High Energy Chemistry, 2005, vol. 39(3), pp. 175-178. DOI: 10.1007/s10733-005-0035-0
Шишканов Олег Николаевич - аспирант кафедры оптоэлектроники, Кубанский государственный университет, Краснодар, Российская Федерация; e-mail: shishkanovoleg@mail.ru. ORCID iD 0000-0001-5373-871X.

Бойченко Александр Павлович - д. ф.-м. н., доцент, Кубанский государственный университет, Краснодар, Российская Федерация; e-mail: bojchenco@yandex.ru. ORCID iD 0000-0001-57505200 .
Shishkanov Oleg N. - postgraduate student, Kuban State University, Krasnodar, Russian Federation; e-mail: shishkanovoleg@mail.ru. ORCID iD 0000-0001-5373-871X.

Boychenko Aleksandr P. - Dr. Sci. (Phys.Math.), Associate Professor, Kuban State University, Krasnodar, Russian Federation; e-mail: bojchenco@yandex.ru. ORCID iD 0000-0001-57505200 . 